\title{
ОСОБЛИВОСТІ МОДЕЛЮВАННЯ ЦІНОВОЇ ДИНАМІКИ НА ОСНОВІ МОДЕЛІ СЕV
}

\section{FEATURES OF PRICE DYNAMICS MODELING BASED ON CEV MODEL}

\author{
Янішевський Василь Степанович \\ кандидат фрізико-математичних наук, доцент, \\ Національний університет «Львівська політехніка» \\ ORCID: https://orcid.org/0000-0002-0449-6686 \\ Фульмес Юрій Ігорович \\ магістр, \\ Національний університет «Львівська політехніка» \\ ORCID: https://orcid.org/0000-0001-6621-6718 \\ Yanishevskyi Vasyl, Fulmes Yuri \\ Lviv Polytechnic National University
}

Розглянуто застосування відомої стохастичної моделі постійної еластичності дисперсії (CEV) до визначення ціни опціону. Знайдена густина умовної ймовірності випадкової величини (ціни активу) моделі для довільного значення параметра еластичності дисперсії $\beta$. Показано, що в залежності від параметра $\beta$ існують два розв'язки для густини умовної ймовірності, які нормовані на одиницю. Один з них належить додатній області зміни параметра $\beta$, інший - від'ємній області зміни $\beta$. Для $\beta<0$ вказаний розв'язок описує лише інтервал $-1<\beta<-\frac{1}{2}$. Визначені ціна європейського опціону кол для значення параметра $\beta=-\frac{1}{2}$ моделі для двох зазначених розв'язків густини умовної ймовірності. При цьому використано також розв'язок для густини умовної ймовірності процесу Феллера, який в границі співпадає з моделлю CEV для $\beta=-\frac{1}{2}$. Проведено порівняль-
ний аналіз чисельних розрахунків цін опціону.

Ключові слова: стохастичне рівняння, броунівський рух, модель геометричного броунівського руху, модель CEV, рівняння Фоккера-Планка, стохастичний процес Феллера, ціна опціону.

Рассмотрено применение известной стохастической модели постоянной эластичности дисперсии (CEV) к определению цены опциона. Найдена плотность условной вероятности случайной величины (цены актива) модели для произвольного значения параметра эластичности дисперсии $\beta$. Показано, что в зависимости от параметра $\beta$ существуют два решения для плотности условной вероятности, которые нормированные на единицу. Один из них принадлежит положительной области изменения параметра $\beta$, другой - отрицательной области изменения $\beta$. Для $\beta<0$ указанный решение описывает только интервал $-1<\beta<-\frac{1}{2}$. Определены цена европейского опциона колл для значения параметра $\beta=-\frac{1}{2}$ модели для двух указанных решений плотности условной вероятности. При этом использовано также решение для плотности условной вероятности процесса Феллера, который в пределе совпадает с моделью CEV для $\beta=-\frac{1}{2}$. Проведен сравнительный
анализ численных расчетов цен опциона.

Ключевые слова: стохастическое уравнение, броуновское движение, модель геометрического броуновского движения, модель CEV, уравнение Фоккера-Планка, стохастический процесс Феллера, цена опциона.

A known constant elasticity of variance (CEV) option pricing model is investigated for the purpose of determining stochastic price dynamics of assets and option price. The CEV model is an attempt to generalize the geometric Brownian motion of Black-Scholes model. As it is known the stochastic dynamics of stock price (asset) that is defined by geometric Brownian motion is quite logical, however it doesn't take into account dispersion change, and assumes it to be constant. The peculiarity of the CEV model is that according to it the volatility changes according to the base price which aligns with theoretical and a lot of empirical data. Because of that the CEV pricing model is considered 
an important step in the Black-Scholes model evolution which allows to cover in one way all other known stochastic models depending on values of parameter $\beta$. However it was found out that for the density of transition probability the dynamics of which is given by CEV model, one should use different solutions based on positive and negative values of parameter $\beta$. The solution which is normalized by a unit for positive values of parameter $\beta$, is different for negative values of parameter $\beta$. Despite this in works of many authors a single solution is used for the entire domain of parameter $\beta$. In the work a transition probability density of stochastic variable (asset price) was found for model with arbitrary value of elasticity density parameter $\beta$. Depending on value of parameter $\beta$ two solutions for transitional probability density were defined. One solution describe a positive domain of parameter $\beta$, the other - negative domain of $\beta$. In addition it was shown that the given solution for $\beta<0$ describes only interval $-1<\beta<-\frac{1}{2}$. The parameter domain $-\frac{1}{2}<\beta<0$ needs separate research. A detailed research of pricing in CEV model for $\beta=-\frac{1}{2}$ was carried out. Pricing of European call option for a given parameter $\beta$ was defined for a model based on two given solutions for transition probability density. The solution for transition probability density of Feller process was used, which in limit matches the CEV model for $\beta=-\frac{1}{2}$. A comparative analysis of numeric calculations for option price
was carried out.

Keywords: stochastic equations, Brownian motion, model of geometric Brownian motion, CEV model, Fokker Planck equation, Feller model, option price.

Постановка проблеми. Блек - Шоулза [1] вивели відому фрормулу ціноутворення опціонів, вважаючи, що ціна базової акції описується геометричним броунівським рухом. Стохастична динаміка цін на акції (активи) $€$ доволі логічною, проте не враховує зміну дисперсії з часом, а вважає ії̈ сталою. Однак, загалом, статистичні закономірності такі як: ефект важеля [2], усмішка волатильності [3] не узгоджуються з логнормальним розподілом і сталою волатильністю.

Для вирішення цієї проблеми були запропоновані альтернативні стохастичні процеси, такі як модель Мертона, модель Кокса Росса та ряд інших моделей [4]. Модель CEV (constant elasticity of variance) $€$ одним із способів узагальнити геометричний броунівський рух моделі Блек - Шоулза. Особливістю моделі CEV $€$ також те, що у ній волатильність змінюється із базовою ціною, що узгоджується як 3 теоретичними аргументами, так i багатьма емпіричні даними [4]. Тому модель ціноутворення CEV розглядається як важливий крок розвитку моделі ціноутворення Блек - Шоулза.

Аналіз останніх досліджень і публікацій. Як ми вже зазначали, модель БлекШоулза [1] визначає постійну волатильність ціни акції, що не узгоджується 3 деякими емпіричними закономірностями. Через те були запропоновані різні узагальнення моделі Блека - Шоулза: моделі із часовою залежністю волатильності, стохастичної волатильності [5]. У випадку стохастичної волатильності актив і волатильність описуються власними динамічними процесами. Найбільш відомою із моделей стохастичної волатильності $€$ модель Гестона [6].
В моделях іншого типу лише динаміка ціни базового активу описується стохастичним процесом, а волатильність задається залежною від величини ціни базового активу. До таких належить модель CEV, що була запропонована Коксом $[7 ; 8]$. Модель CEV неперервним чином охоплює відомі стохастичні процеси - геометричний броунівський рух, модель Кокса - Роса, модель Орнштейна - Уленбека, які отримуються для різних значень параметра $\beta$ моделі (1).

Відзначається, що модель CEV порівняно 3 моделлю Блек - Шоулза має переваги у прогнозуванні як цін активів, так і опціонів на них [9]. Крім того аналіз на основі моделі CEV $€$ простіший порівняно $з$ більшістю моделей стохастичної волатильності, її значно простіше реалізувати та калібрувати [10]. Поряд 3 цим точна формула ціноутворення опціону за моделлю CEV подається у вигляді нескінченного ряду неповних гамма-функцій [7], аналіз якого $€$ непростим. Розглядалась також модель типу CEV, де дрейср і волатильність містять параметри залежні від часу [11], аналізувалось рівняння для ціни опціону.

Постановка задачі. По при те, що модель CEV досліджувалась в роботах багатьох авторів, $€$ ряд не розв'язаних задач. Це, зокрема, стосується розв'язків для густини умовної ймовірності випадкової величини, що задана рівнянням (1). В багатьох роботах розв'язки справедливі для області значень параметра моделі $\beta>0$ застосовуються також для $\beta<0$, де вони $€$ не нормованими на одиницю. Тому В даній роботі досліджується густина ймовірності для рівняння CEV в залежності від параметра $\beta$, визначені межі застосовності вказаних розв'язків та досліджується ціна європейського опціону кол. 


\section{Виклад основного матеріалу.}

Моделювання цінової динаміки на основі стохастичного рівняння CEV

Модель CEV визначає цінову динаміку активу таким стохастичним рівнянням

$$
d S(t)=\mu S(t) d t+\sigma S(t)^{1+\beta} d w(t),
$$

де $S(t)$ - випадкова величина, яка описує ціну активу, $\mu>0$ - має зміст відсоткової ставки, $\sigma>0$ - множник волатильності, $d w(t)$ - вінерівський процес 3 характеристиками $d w(t)=0, d w(t)^{2}=d t(\langle\ldots\rangle$ позначає усереднення за всіма реалізаціями стохастичного процесу), $\beta$ - параметр, що визначає еластичність волатильності.

Модель CEV (1) $є$ узагальненням ряду відомих стохастичних моделей. Зокрема для $\beta=0$ отримаємо модель геометричного броунівського руху [6]. У випадку $\beta=-\frac{1}{2}$ отримаємо модель Кокса-Роса (процес квадратного корня), яка була запропонована в [12]. Значення $\beta=-1$ відповідає моделі Орнштейна Уленбека, яка в фрінансових задачах відома також як модель Васічека [4]. Через те модель (1) розглядають для значень $-1<\beta<0$, де параметр $\beta$ змінюється неперервно на цьому інтервалі.

3 рівняння (1) знайдемо, що миттєва волатильність процесу рівна $\sigma^{2} S(t)^{2 \beta}$. Відповідно логарисрмічна похідна від неї (еластичність волатильності) дорівнює

$$
d \ln \left(\sigma^{2} S(t)^{2 \beta}\right) / d \ln S(t)=2 \beta
$$

і не залежить від значення випадкової змінної $S(t)$. 3 цією властивістю власне і пов'язана назва моделі (1).

Як ми вже зазначали, модель CEV використовується для опису стохастичної цінової динаміки базових активів і ціноутворення деривативів, опціонів зокрема. В роботі [13] досліджувались розв'язки рівняння БлекаШоулза для ціни опціону на основі стохастичного рівняння (1). В роботах $[14 ; 15]$ розглядався випадок Кокса-Роса ( $\left.\beta=-\frac{1}{2}\right)$ [12].

Відомо [4], що різні показники орінансової інженерії можна визначити, якщо задана густина умовної ймовірності $K\left(S, S_{0}, t, t_{0}\right)$ випадкової величини, що описується стохастичним рівнянням (1). Тут $S$ і $S_{0}$ - визначають значення випадкової величини $S(\tau)$ в моменти часу $t$ i $t_{0}\left(t>t_{0}\right)$. Рівняння (1) $є$ частковим випадком загального стохастичного рівняння вигляду

$$
d S(t)=A(S(t)) d t+B(S(t)) d w(t),
$$

де $A(S(t))$ і $B(S(t))$ - дрейср і волатильність стохастичного процесу відповідно.

Густину умовної ймовірності $K\left(S, S_{0}, t, t_{0}\right)$ стохастичного процесу (3), як правило, визначають на основі рівняння Фоккера-Планка [16]. В даній роботі використано еквівалентний спосіб - густину умовної ймовірності для процесу (3) виразимо фрункціональним інтегралом [17]

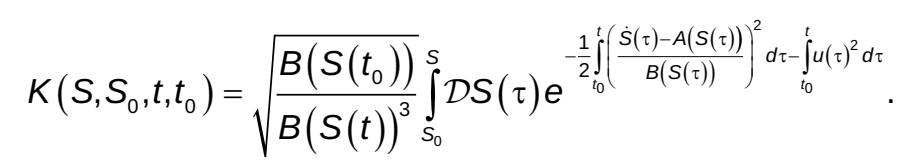

У фрормулі (4) позначені величини:

$$
\begin{gathered}
u(\tau)=\frac{1}{2} A^{\prime}(S(\tau))-A(S(\tau)) \frac{B^{\prime}(S(\tau))}{B(S(\tau))}+\frac{1}{8} B^{\prime}(S(\tau))^{2}-\frac{1}{4} B(S(\tau)) B^{\prime \prime}(S(\tau)), \\
\mathcal{D S}(\tau)=\prod_{\tau} \frac{d S(\tau)}{\sqrt{2 \pi B(S(\tau))^{2} d \tau}} .
\end{gathered}
$$

Знак «штрих» біля величин у формулі (5) вказує на похідну за аргументом.

Підставляючи 3 рівняння (1) значення $A(S(t))=\mu S(t), \quad B(S(t))=\sigma S(t)^{1+\beta} \quad$ отримаємо густину ймовірності для моделі CEV у вигляді фрункціонального інтегралу

$$
\begin{gathered}
K_{a}\left(S, S_{0}, t, t_{0}\right)=S^{-\frac{3}{2}(1+\beta)} S_{0}^{\frac{1}{2}(1+\beta)} e^{\frac{1}{2}(1+2 \beta)(t-t 0)} \times \\
\int_{S_{0}}^{S} \mathcal{D} \tilde{S}(\tau) e^{-\frac{1}{2 \sigma^{2}} \int_{t_{0}}^{t} S(\tau)^{-2(1+\beta)}\left(\dot{S}(\tau)-\mu_{0} S(\tau)\right)^{2} d \tau-\frac{\sigma^{2}}{8}\left(1-\beta^{2}\right) \int_{t_{0}}^{t} S(\tau)^{2 \beta} d \tau}, \\
\mathcal{D S}(\tau)=\prod_{\tau} \frac{d S(\tau)}{\sqrt{2 \pi \sigma^{2} S(\tau)^{2(1+\beta)} d \tau}} .
\end{gathered}
$$

Функціональний інтеграл у фрормулі (6) зведемо до відомого [18] здійснюючи заміну змінних $y(t)=-\frac{S(t)^{-\beta}}{\beta}$

$$
\begin{gathered}
K_{a}\left(S, S_{0}, t, t_{0}\right)=S^{-\frac{3}{2}(1+\beta)} S_{0}^{\frac{1}{2}(1+\beta)} e^{\frac{1}{2}(1+2 \beta)(t-t 0)} \times \\
\int_{y_{0}}^{y} \mathcal{D} y(\tau) e^{-\frac{1}{2 \sigma^{2}} \int_{t_{0}}^{t} y(\tau)^{2} d \tau-\frac{1}{2} \beta^{2} \mu^{2} \int_{t_{0}}^{t} y(\tau)^{2} d \tau-\frac{1}{8}\left(\frac{1}{\left(\beta^{2}-1\right.}\right)_{t_{0}}^{t} \frac{d \tau}{y(\tau)^{2}},} \\
\mathcal{D} y(\tau)=\prod_{\tau} \frac{d y(\tau)}{\sqrt{2 \pi d \tau}}, \\
\text { де позначено } y=\frac{S^{-\beta}}{|\beta| \sigma}, y_{0}=\frac{S_{0}^{-\beta}}{|\beta| \sigma} .
\end{gathered}
$$


Для фрункціонального інтегралу (7) відома точна фрормула [18], в результаті отримаємо

$$
\begin{aligned}
& K_{a}\left(S, S_{0}, t, t_{0}\right)=e^{\frac{1}{2}(1+2 \beta)(t-t 0)} \frac{\mu S^{\frac{3}{2}-2 \beta} S_{0}^{\frac{1}{2}}}{\sigma^{2} \operatorname{sign}(\beta) \sinh \left(\beta \mu\left(t-t_{0}\right)\right)} \times \\
& e^{-\frac{\mu S^{-2 \beta}}{2 \beta \sigma^{2}}\left(1+\operatorname{coth}\left(\beta \mu\left(t-t_{0}\right)\right)\right)+\frac{\mu S_{0}^{-2 \beta}}{2 \beta \sigma^{2}}\left(1-\operatorname{coth}\left(\beta \mu\left(t-t_{0}\right)\right)\right)} I_{\frac{1}{2|\beta|}}\left(\frac{\mu \cdot\left(S S_{0}\right)^{-\beta}}{\beta \sigma^{2} \sinh \left(\beta \mu\left(t-t_{0}\right)\right)}\right) \text {, } \\
& I_{v}(z)=\sum_{k=0}^{\infty} \frac{\left(\frac{z}{2}\right)^{2 k+v}}{k ! \Gamma(k+v+1)}, \\
& \frac{\partial C\left(S_{0}, t, t_{0}\right)}{\partial t_{0}}+\frac{\sigma^{2} S_{0}^{2(1+\beta)}}{2} \frac{\partial^{2} C\left(S_{0}, t, t_{0}\right)}{\partial S_{0}^{2}}+ \\
& +\mu \frac{\partial\left(S K_{a}\left(S, S_{0}, t, t_{0}\right)\right)}{\partial S}=0 .
\end{aligned}
$$

$\Gamma(k)$ - гамма фрункція.

Метод фрункціонального інтегралу Фейнмана застосовувався для аналізу моделі CEV також у [20], проте використовувався наближений метод розрахунку. На відміну від [20] фоормула (8) задає точний вираз для густини умовної ймовірності моделі.

Густина умовної ймовірності (8) співпадає 3 такою знайденою на основі розв'язку диференціального рівняння Фоккера-Планка [21]. Безпосередньою підстановкою можна переконатись також, що $K_{a}\left(S, S_{0}, t, t_{0}\right)$ задовольняє рівнянню Фоккера-Планка

$$
\begin{gathered}
\frac{\partial K_{a}\left(S, S_{0}, t, t_{0}\right)}{\partial t}-\frac{\sigma^{2}}{2} \frac{\partial^{2}\left(S^{2(1+\beta)} K_{a}\left(S, S_{0}, t, t_{0}\right)\right)}{\partial S^{2}}+ \\
+\mu \frac{\partial\left(S K_{a}\left(S, S_{0}, t, t_{0}\right)\right)}{\partial S}=0 .
\end{gathered}
$$

Як відомо [4] ціна європейського опціону кол визначається усередненням платіжної фрункції $F(S)=(S-K)^{+}$, де $F(S)>0$, для $S>K$, і $F(S)=0$ для $0<S<K, K$ - ціна виконання опціону. Отже, ціну опціону визначаємо таким інтегралом

$$
C\left(S_{0}, t, t_{0}\right)=e^{-\mu\left(t-t_{0}\right)} \int_{0}^{\infty} K_{a}\left(S, S_{0}, t, t_{0}\right) F(S) d S .
$$

Зауважимо, що величина $e^{\mu\left(t-t_{0}\right)} C\left(S_{0}, t, t_{0}\right)$ згідно (10) задовольняє оберненому рівнянню Колмогорова [16]. На основі цього отримуємо рівняння ціни опціону $C\left(S_{0}, t, t_{0}\right)$

Розв'язки рівняння для ціни опціону (11) досліджувались в роботі [13].

Як було зазначено, важливим для практичного застосування $\epsilon$ випадок $\beta<0$, для якого миттєва волатильність процесу $\left(\sigma^{2} S(t)^{2 \beta}\right)$ зменшується із зростанням ціни активу $S$. Проте з'ясувалось, що розв'язки для густини умовної ймовірності, що використовувались у роботах $[7 ; 13 ; 14 ; 21]$ для $\beta<0$ не задовольняють умову нормування. Зокрема, для інтегралу знайдемо

$$
\begin{gathered}
\int_{0}^{\infty} K_{a}\left(S, S_{0}, t, t_{0}\right) d S= \\
=1-\frac{\Gamma\left(\frac{1}{2|\beta|}, \frac{\mu S_{0}^{2 \beta \mid}}{\beta \sigma^{2}\left(1-e^{-2 \beta \mu\left(t-t_{0}\right)}\right)}\right)}{\Gamma\left(\frac{1}{2|\beta|}\right)}<1,
\end{gathered}
$$

де позначені $\Gamma(x), \Gamma(a, x)$ - гамма-срункція i неповна гамма-фуункція відповідно [19].

Як видно, $K_{a}\left(S, S_{0}, t, t_{0}\right)$ нормована на одиницю лише в границі $t \rightarrow t_{0}$. Зрозуміло, що така особливість розв'язку впливатиме на ціну опціону визначену за фрормулою (10).

Можна переконатись, що рівнянню Фоккера-Планка (9) задовольняє також розв'язок, який отримаємо з (8) заміною у функції Бесселя $I_{\frac{1}{2|\beta|}}(x) \rightarrow I_{-\frac{1}{2|\beta|}}(x)$, а саме

$$
\begin{gathered}
K_{b}\left(S, S_{0}, t, t_{0}\right)=e^{\frac{1}{2}(1+2 \beta)\left(t-t_{0}\right)} \frac{\mu S^{-\frac{3}{2} 2 \beta} S_{0}^{\frac{1}{2}}}{\sigma^{2} \operatorname{sign}(\beta) \sinh \left(\beta \mu\left(t-t_{0}\right)\right)} \times \\
e^{-\frac{\mu S^{-2 \beta}}{2 \beta \sigma^{2}\left(1+\operatorname{coth}\left(\beta \mu\left(t-t_{0}\right)\right)\right)+\frac{\mu S_{0}^{-2 \beta}}{2 \beta \sigma^{2}}\left(1-\operatorname{coth}\left(\beta \mu\left(t-t_{0}\right)\right)\right)} I_{\frac{1}{2|\beta|}}\left(\frac{\mu \cdot\left(S S_{0}\right)^{-\beta}}{\beta \sigma^{2} \sinh \left(\beta \mu\left(t-t_{0}\right)\right)}\right)}
\end{gathered}
$$

Обидва розв'язки (8) і (13) прямують до нуля $K_{a, b}\left(S, S_{0}, t, t_{0}\right) \rightarrow 0$, проте виявляють різну поведінку для $s \rightarrow 0^{+}$. Введемо позна- чення $\beta=-\beta_{0}, \beta_{0}>0$. Тоді характер залежності $K_{a}\left(S, S_{0}, t, t_{0}\right)(8)$ в околі точки $S \rightarrow 0^{+}$задається множником $S^{-1+2 \beta_{0}}$. Для розв'язку $K_{b}\left(S, S_{0}, t, t_{0}\right)$ 
характер асимптотики буде таким $-S^{2\left(\beta_{0}-1\right)}$. Звідси видно, що інтеграл $\int_{0}^{\infty} K_{a}\left(S, S_{0}, t, t_{0}\right) d S$ існує для усіх $\beta_{0}>0$, а $\int_{0}^{\infty} K_{b}\left(S, S_{0}, t, t_{0}\right) d S$ лише для $\beta_{0}>\frac{1}{2}$. Можна також переконатись, що $\int_{0}^{\infty} K_{b}\left(S, S_{0}, t, t_{0}\right) d S=1$ для $-1<\beta<-\frac{1}{2}$, тобто виконується умова нормування на одиницю.

Таким чином, області значень параметра $0<\beta<1$ відповідає густина умовної ймовірності $K_{a}\left(S, S_{0}, t, t_{0}\right)$, області зміни параметра $-1<\beta<-\frac{1}{2}$ відповідає $-K_{b}\left(S, S_{0}, t, t_{0}\right)$. Випадок, де $-\frac{1}{2}<\beta<0$ вимагає окремого дослідження, оскільки розв'язок $K_{a}\left(S, S_{0}, t, t_{0}\right)$ у цій області не нормований на одиницю, а для розв'язку $K_{b}\left(S, S_{0}, t, t_{0}\right)$ відповідний інтеграл не існує.

Аналіз моделі CEV для $\beta=-\frac{1}{2}$

Розглянемо більше детально стохастичне рівняння для $\beta=-\frac{1}{2}$

$$
d S(t)=\mu S(t) d t+\sigma \sqrt{S(t)} d w(t) .
$$

Вперше дане рівняння було запропоноване для опису ціноутворення активів і деривативів в роботі [12]. Для густини умовної ймовірності був використаний розв'язок $K_{a}\left(S, S_{0}, t, t_{0}\right)$ (8) для $\beta=-\frac{1}{2}$, проте він не задовольняє

$$
\begin{aligned}
& K_{F}\left(S, S_{0}, t, t_{0}\right)=\frac{\mu}{\sigma^{2} \sinh \frac{1}{2} \mu\left(t-t_{0}\right)} e^{-\frac{\mu^{2} \alpha\left(t-t_{0}\right)}{\sigma^{2}}} e^{\frac{\mu\left(S-S_{0}\right)}{\sigma^{2}}} e^{-\frac{\mu\left(S+S_{0}\right) \operatorname{coth} \frac{1}{2} \mu\left(t-t_{0}\right)}{\sigma^{2}}} \times\left(\frac{S}{S_{0}}\right)^{-\frac{1}{2}+\frac{\alpha \mu}{\sigma^{2}}} I_{-1+\frac{\alpha \alpha \mu}{\sigma^{2}}}\left(\frac{2 \mu \sqrt{S S_{0}}}{\sigma^{2} \sinh \frac{1}{2} \mu\left(t-t_{0}\right)}\right) . \\
& C_{F}\left(S_{0}, t, t_{0}\right)=e^{-\mu\left(t-t_{0}\right)}(S-K)+ \\
& e^{-\mu\left(t-t_{0}\right)} \int_{0}^{K} K_{F}\left(S, S_{0}, t, t_{0}\right)(K-S) d S .
\end{aligned}
$$

$$
\int_{0}^{\infty} K_{F}\left(S, S_{0}, t, t_{0}\right) d S=1 .
$$

Перші моменти випадкової величини $S$ рівні:

$$
\begin{gathered}
S=\int_{0}^{\infty} K_{F}\left(S, S_{0}, t, t_{0}\right) S d S=\alpha\left(e^{\mu\left(t-t_{0}\right)}-1\right)+S_{0} e^{\mu\left(t-t_{0}\right)}, \\
S^{2}=\int_{0}^{\infty} K_{F}\left(S, S_{0}, t, t_{0}\right) S^{2} d S=S^{2}+ \\
\frac{\sigma^{2}}{2 \mu}\left(e^{\mu\left(t-t_{0}\right)}-1\right)\left(\alpha\left(e^{\mu\left(t-t_{0}\right)}-1\right)+2 S_{0} e^{\mu\left(t-t_{0}\right)}\right) .(18)
\end{gathered}
$$

В границі $\alpha \rightarrow 0$ середні значення $S, S^{2}$ для процесу Феллера співпадають 3 такими знайденими на основі $K_{a}\left(S, S_{0}, t, t_{0}\right)$ $K_{b}\left(S, S_{0}, t, t_{0}\right)(15)$ для $\beta=-\frac{1}{2}$.

Запишемо ціну опціону кол з використанням стохастичного процесу Феллера у такому вигляді умову нормування. Натомість ми розглянемо розв'язок (13) для $\beta=-\frac{1}{2}$, густина умовної ймовірності у цьому випадку має вигляд

$$
\begin{aligned}
K_{b}\left(S, S_{0}, t, t_{0}\right) & =\frac{\mu}{\sigma^{2} \sinh \frac{1}{2} \mu\left(t-t_{0}\right)} e^{\frac{\mu\left(S-S_{0}\right)}{\sigma^{2}}} e^{-\frac{\mu\left(S+S_{0}\right) \operatorname{coth} \frac{1}{2}\left(t\left(t-t_{0}\right)\right.}{\sigma^{2}}} \times \\
& \left(\frac{S}{S_{0}}\right)^{-\frac{1}{2}} I_{-1}\left(\frac{2 \mu \sqrt{S S_{0}}}{\sigma^{2} \sinh \frac{1}{2} \mu\left(t-t_{0}\right)}\right)
\end{aligned}
$$

Розв'язок (15) слід розглядати в границі $\varepsilon \rightarrow 0 \quad\left(\beta=-\frac{1}{2}-\varepsilon, \varepsilon>0\right)$, щоб забезпечити збіжність інтегралу $\int_{0}^{\infty} K_{b}\left(S, S_{0}, t, t_{0}\right) d S$. Ми використаємо еквівалентний спосіб, де стохастичне рівняння (14) будемо розглядати як частковий випадок процесу Феллера $(\alpha>0)$

$$
d S(t)=\mu(S(t)+\alpha) d t+\sigma \sqrt{S(t)} d w(t) .
$$

Застосовуючи в кінцевих фрормулах границю $\alpha \rightarrow 0$, отримаємо опис на основі стохастичного рівняння (14).

Густину умовної ймовірності процесу Феллера визначимо на основі фрормул (4) і (5), в результаті знайдемо
Для рівняння (14) 3 густиною ймовірності $K_{a}\left(S, S_{0}, t, t_{0}\right)(8)\left(\beta=-\frac{1}{2}\right)$ отримаємо для ціни
опціону

$$
\begin{aligned}
& C_{a}\left(S_{0}, t, t_{0}\right)=e^{-\mu\left(t-t_{0}\right)}(S-K)+e^{-\mu\left(t-t_{0}\right)} K e^{-\frac{2 \mu S_{0}}{\sigma^{2}\left(1-e^{-\mu\left(t-t_{0}\right)}\right)}}+ \\
& e^{-\mu\left(t-t_{0}\right)} \int_{0}^{K} K_{a}\left(S, S_{0}, t, t_{0}\right)(K-S) d S .
\end{aligned}
$$

Другий доданок у фрормулі (20) пов'язаний 3 тим, що густина умовної ймовірності $K_{a}\left(S, S_{0}, t, t_{0}\right)$ не нормована на одиницю (12).

Інтеграли, які містяться у наведених фрормулах, доволі складні для розрахунків. Як правило, для них отримують співвідношення у вигляді рядів, підставляючи для відповідних фрункцій Бесселя розвинення у ряд (див. фрормулу (8)). Для чисельних розрахунків ми 
використаємо наведені вище фрорми запису у вигляді інтегралів.

Спершу виконаємо деякі перетворення в інтегралі у (19). Це пов'язано з тим, що границю $\alpha \rightarrow 0$ не можна зразу виконати у (19) через розбіжність у доданку $\int_{0}^{K} K_{F}\left(S, S_{0}, t, t_{0}\right) K d S$ в околі $S \sim 0^{+}$. Тому в інтегралі (19) виконаємо інтегруванням за частинами і запишемо інтеграл у вигляді $\int_{0}^{k} K_{F}\left(S, S_{0}, t, t_{0}\right)(K-S) d S=\int_{0}^{k} u(S) d v(S)=\int_{0}^{k} u^{\prime}(S) V(S) d S$,

$$
\begin{gathered}
u(S)=\frac{\mu}{\sigma^{2} \sinh \frac{1}{2} \mu\left(t-t_{0}\right)} e^{-\frac{\mu^{2} \alpha\left(t-t_{0}\right)}{\sigma^{2}}} e^{\frac{\mu\left(S-S_{0}\right)}{\sigma^{2}}} e^{-\frac{\mu\left(S+S_{0}\right) \operatorname{coth} \frac{1}{2} \mu\left(t-t_{0}\right)}{\sigma^{2}}}(K-S), \\
d v(S)=\left(\frac{S}{S_{0}}\right)^{-\frac{1}{2}+\frac{\alpha \mu}{\sigma^{2}}} I_{-1+\frac{2 \alpha \mu}{\sigma^{2}}}\left(\frac{2 \mu \sqrt{S S_{0}}}{\sigma^{2} \sinh \frac{1}{2} \mu\left(t-t_{0}\right)}\right) d S,
\end{gathered}
$$$$
\left.V(S)=\frac{\sigma^{2}}{\mu}\left(\frac{S}{S_{0}}\right)^{\frac{\alpha \mu}{\sigma^{2}}} \sinh \frac{1}{2} \mu\left(t-t_{0}\right) \frac{I_{\frac{2 \alpha \mu}{\sigma^{2}}}}{\left(\sigma^{2} \sinh \frac{1}{2} \mu\left(t-t_{0}\right)\right.}\right) \text {. }
$$

В перетвореному інтегралі в (21) виконаємо граничний перехід $\alpha \rightarrow 0$. В результаті після ряду перетворень отримаємо вираз для ціни опціону у випадку стохастичного процесу (14), густина умовної ймовірності для якого задана фрормулою (8) для $\beta=-\frac{1}{2}$

$$
\begin{gathered}
\tilde{C}_{b} / S_{0}=1-x e^{-\tau}+\int_{0}^{1} \Delta C_{b}(S) d S \\
\Delta C_{b}(S)=x e^{-2 \rho_{0} \frac{e^{\tau}+S x}{e^{\tau}-1}}\left(1+2 x \rho_{0} \frac{1-S}{e^{\tau}-1}\right) I_{0}\left(\frac{2 \rho_{0} \sqrt{x S}}{\sinh \frac{1}{2} \tau}\right) .
\end{gathered}
$$

Тут введені позначення $\rho_{0}=\frac{S_{0} \mu}{\sigma^{2}}, \tau=\mu T$, $T=\left(t-t_{0}\right), K=S_{0} x$.

Подібним чином запишемо вираз для ціни опціону (20)

$$
\begin{gathered}
\tilde{C}_{a} / S_{0}=1-x e^{-\tau}\left(1-e^{-\frac{2 \rho_{0}}{1-e^{-\tau}}}\right)+\int_{0}^{1} \Delta C_{a}(S) d S, \\
\Delta C_{a}(S)=x^{\frac{3}{2}} e^{-2 \rho_{0} \frac{e^{\tau}+S x}{e^{\tau}-1}} \frac{\rho_{0}(1-S)}{\sqrt{S} \sinh \frac{1}{2} \tau} l_{1}\left(\frac{2 \rho_{0} \sqrt{x S}}{\sinh \frac{1}{2} \tau}\right) .
\end{gathered}
$$

На основі виразів (22) і (23) виконаємо чисельні розрахунки значення ціни опціону для деяких значень параметрів. Для цього, зокрема, скористаємось значеннями вхідних параметрів для моделі, які наведені у роботі [22]. Дані чисельних розрахунків цін наведені у таблиці 1.

Формули (22) і (23) свідчать про нелінійні залежності цін опціонів від параметрів моделі і значень $S_{0}, K$. З таблиці видно, що ціни опціонів знайдені для різних розв'язків густин умовних ймовірностей $K_{a}\left(S, S_{0}, t, t_{0}\right)$ і $K_{b}\left(S, S_{0}, t, t_{0}\right)$ мало різняться для певних значень вхідних параметрів. Помітні відхилення виникають із зростанням T, $\sigma$ і зменшенням SO.

Висновки. Розглянута модель CEV для опису ціноутворення активів та деривативів, опціонів зокрема. Дослідження проведено на основі густини умовної ймовірності випадкової змінної рівняння (1), яка моделює динаміку ціни активу (акції). Методом фрункціонального інтегралу знайдені розв'язки для густини умовної ймовірності вказаної випадкової величини. Показано, що існують два розв'язки, кожен з яких застосовний для значень параметра $\beta>0$ чи $\beta<0$ відповідно. Для від'ємних значень $\beta<0$ розв'язок визначений лише на інтервалі $-1<\beta<-\frac{1}{2}$. Таким чином для зазначених областей параметрів слід використовувати різні розв'язки, а не один, як прийнято в багатьох роботах.

Детально розглянуто випадок моделі CEV з $\beta=-\frac{1}{2}$. У цьому випадку модель CEV можна також отримати як граничний випадок стохастичного процесу Феллера, що більш зручно для розрахунків. Для розрахунків ціни опціону вико-

Таблиця 1

Результати чисельних розрахунків цін опціонів за формулами (22) і (23)

\begin{tabular}{|c|c|c|c|c|c|c|c|}
\hline № & $\boldsymbol{S}_{0}$ & $K$ & $\boldsymbol{\mu}$ & $\boldsymbol{\sigma}$ & $\boldsymbol{T}$ & $\tilde{\boldsymbol{C}}_{\boldsymbol{b}} / \boldsymbol{S}_{\mathbf{0}}$ & $\tilde{\boldsymbol{C}}_{\boldsymbol{a}} / \boldsymbol{S}_{\mathbf{0}}$ \\
\hline 1 & 2 & 2 & 0,01 & 0,14 & 1 & 0,045 & 0, \\
\hline 2 & 2 & 2 & 0,18 & 0,42 & 1 & 0,22 & 0,22 \\
\hline 3 & 2 & 2 & 0,013 & 0,35 & 2 & 0,154 & 0,154 \\
\hline 4 & 2 & 2 & 0,05 & 0,72 & 1 & 0,231 & 0,231 \\
\hline 5 & 2,1 & 2 & 0,05 & 0,72 & 1 & 0,25 & 0,25 \\
\hline 6 & 2 & 2 & 0,05 & 0,72 & 2 & 0,343 & 0,341 \\
\hline 7 & 1,5 & 2 & 0,05 & 0,72 & 3 & 0,40 & 0,37 \\
\hline 8 & 1,5 & 2 & 0,1 & 0,72 & 4 & 0,62 & 0,65 \\
\hline
\end{tabular}


ристано густину умовної ймовірності процесу Феллера. В результаті відповідної границі отримано опис на основі моделі CEV $3 \beta=-\frac{1}{2}$. Наведена також ціна опціону у випадку густини умовної ймовірності, що пов'язана з ненормованим на одиницю розв'язком. Проведено чисельний порівняльний аналіз для ряду вхідних параметрів моделі, де показано, що значення цін опціону відрізняються із зростанням значень параметрів $T, \sigma$, а також суттєво залежать від початкової ціни активу $\mathrm{S}_{0}$.

\section{СПИСОК ВИКОРИСТАНИХ ДЖЕРЕЛ:}

1. Fischer Black and Myron Scholes. The pricing of options and corporate liabilities. Journal of Political Economy, 81(3):637-654, 1973.

2. Tim Bollerslev, Julia Litvinova, and George Tauchen. Leverage and volatility feedback effects in highfrequency data. Journal of Financial Econometrics, 4(3):353-384, 2006.

3. Jens Carsten Jackwerth and Mark Rubinstein. Recovering probability distributions from option prices. The Journal of Finance, 51(5):1611-1631, 1996.

4. Yuh-Dauh Lyuu (2004) Financial Engineering and Computation: Principles, Mathematics, and Algorithms. Cambridge University Press, $648 \mathrm{p}$.

5. Neil Shephard and Torben G Andersen. Stochastic volatility: origins and overview. In Thomas Mikosch, JensPeter Kreiß, Richard A. Davis, and Torben Gustav Andersen, editors, Handbook of Financial Time Series, pages 233-254. Springer, Berlin, Heidelberg, 2009.

6. Fabrice D. Rouah, Steven L. Heston. The Heston Model and its Extensions in Matlab and C\#, + Website. Published by John Wiley \& Sons, Inc., Hoboken, New Jersey, Year: 2013. 411 p.

7. John C Cox. Notes on option pricing i: Constant elasticity of variance diffusions. Working paper, Stanford University, 1975.

8. John C. Cox. The constant elasticity of variance option pricing model. The Journal of Portfolio Management, 23(5):15-17, 1996.

9. VK Singh and N Ahmad. Forecasting performance of constant elasticity of variance model: Empirical evidence from India. International Journal of Applied Economics and Finance, 5(1):87-96, 2011.

10. K.C. Yuen, H. Yang and K.L. Chu. Estimation in the constant elasticity of variance model. British Actuarial Journal, 7(2):275-292, 2001.

11. P. Carr, A. Itkin and D. Muravey. Semi-closed form prices of barrier options in the time dependent CEV and CIR models, Journal of Derivatives, 28 (2020), 26-50.

12. John C. Cox and Stephen A. Ross. The valuation of options for alternative stochastic processes. Journal of financial economics, 3(1-2):145-166, 1976.

13. C. F. Lo and p. H. Yuen. Constant elasticity of variance option pricing model with time-dependent parameters. International Journal of Theoretical and Applied Finance Vol. 3, No. 4 (2000) 661-674.

14. Y. L. Hsua, T. I. Lina, C. F. Leebc. Constant elasticity of variance (CEV) option pricing model: Integration and detailed derivation. Mathematics and Computers in Simulation. Volume 79, Issue 1, October 2008, Pages 60-71.

15. Stan Beckers. The constant elasticity of variance model and its implications for option pricing. The Journal of Finance, Vol. 35, № 3, p. 661-673, 1980.

16. C. W. Gardiner. Handbook of Stochastic Methods for Physics, Chemistry and the Natural Sciences (2 nd Ed.), Springer-Verlag, New York, 1985.

17. Yanishevsky V. S. Application of the path integral method to some stochastic models of financial engineering. Journal of Physical Studies, 25 (2), 2801: 1-10, 2021.

18.Christian Grosche, Frank Steiner (1998) Handbook of Feynman Path Integrals. Springer Berlin Heidelberg, $444 \mathrm{p}$.

19.Абрамовиц М., Стиган И. Справочник по специальным функциям с формулами, графиками и математическими таблицами. [Пер. с англ.] Москва : «Наука» Главная редакция физико-математической литературы, 1979. 831 с.

20. Axel A. Araneda and Marcelo J. Villena. Computing the CEV option pricing formula using the semiclassical approximation of path integral. arXiv:1803.10376 v1 [q-fin. CP] 28 Mar 2018.

21. Linetsky, V., and R. Mendoza. "Encyclopedia of Quantitative Finance." In Constant Elasticity of Variance (CEV) Diffusion Model. Hoboken, NJ: Wiley \& Sons. 2010.

22. Dan Pirjol and lingjiong Zhu. Short maturity Asian options for the CEV model. Preprint. Arxiv:1702.03382 v1 [q-fin.pr] 11 feb. 2017. 


\section{REFERENCES:}

1. Fischer Black and Myron Scholes (1973) The pricing of options and corporate liabilities. Journal of Political Economy, 81(3):637-654.

2. Tim Bollerslev, Julia Litvinova, and George Tauchen (2006) Leverage and volatility feedback effects in highfrequency data. Journal of Financial Econometrics, 4(3):353-384.

3. Jens Carsten Jackwerth and Mark Rubinstein (1996) Recovering probability distributions from option prices. The Journal of Finance, 51(5):1611-1631.

4. Yuh-Dauh Lyuu (2004) Financial Engineering and Computation: Principles, Mathematics, and Algorithms. Cambridge University Press, $648 \mathrm{p}$.

5. Neil Shephard and Torben G Andersen (2009) Stochastic volatility: origins and overview. In Thomas Mikosch, Jens-Peter Kreiß, Richard A. Davis, and Torben Gustav Andersen, editors, Handbook of Financial Time Series, pages 233-254. Springer, Berlin, Heidelberg.

6. Fabrice D. Rouah, Steven L. Heston (2013) The Heston Model and its Extensions in Matlab and C\#, + Website. Published by John Wiley \& Sons, Inc., Hoboken, New Jersey, Year, 411 p.

7. John C. Cox (1975) Notes on option pricing i: Constant elasticity of variance diffusions. Working paper, Stanford University.

8. John C. Cox (1996) The constant elasticity of variance option pricing model. The Journal of Portfolio Management, 23(5):15-17.

9. V.K. Singh and N. Ahmad (2011) Forecasting performance of constant elasticity of variance model: Empirical evidence from India. International Journal of Applied Economics and Finance, 5(1):87-96.

10. K.C. Yuen, H. Yang and K.L. Chu (2001) Estimation in the constant elasticity of variance model. British Actuarial Journal, 7(2):275-292.

11. P. Carr, A. Itkin and D. Muravey (2020) Semi-closed form prices of barrier options in the time dependent CEV and CIR models, Journal of Derivatives, 28, 26-50.

12. John C. Cox and Stephen A. Ross (1976) The valuation of options for alternative stochastic processes. Journal of financial economics, 3(1-2):145-166.

13. C. F. Lo and p. H. Yuen (2000) Constant elasticity of variance option pricing model with time-dependent parameters. International Journal of Theoretical and Applied Finance, vol. 3, no. 4, 661-674.

14. Y. L. Hsua, T. I. Lina, C. F. Leebc (October 2008) Constant elasticity of variance (CEV) option pricing model: Integration and detailed derivation. Mathematics and Computers in Simulation, vol. 79, issue 1, pp. 60-71.

15. Stan Beckers (1980) The constant elasticity of variance model and its implications for option pricing. The Journal of Finance, vol. 35, no. 3, pp. 661-673.

16. C. W. Gardiner (1985) Handbook of Stochastic Methods for Physics, Chemistry and the Natural Sciences (2 nd Ed.). Springer-Verlag, New York.

17. Yanishevsky V. S. (2021) Application of the path integral method to some stochastic models of financial engineering. Journal of Physical Studies, 25(2), 2801: 1-10.

18. Christian Grosche, Frank Steiner (1998) Handbook of Feynman Path Integrals. Springer Berlin Heidelberg, $444 \mathrm{p}$.

19. Abramovits M., Stigan I. (1979) Spravochnik po spetsial'nym funktsiyam s formulami, grafikami i matematicheskimi tablitsami. [Handbook of mathematical functions with formulas, graphs and mathematical tables]. [Per. s angl.]. Moscow: «Nauka» Glavnaya redaktsiya fiziko-matematicheskoy literatury, 831 p. (in Russian)

20. Axel A. Araneda and Marcelo J. Villena (28 Mar 2018) Computing the CEV option pricing formula using the semiclassical approximation of path integral. arXiv:1803.10376 v1 [q-fin. CP].

21. Linetsky, V., and R. Mendoza (2010) "Encyclopedia of Quantitative Finance." In Constant Elasticity of Variance (CEV) Diffusion Model. Hoboken, NJ: Wiley \& Sons.

22. Dan Pirjol and lingjiong Zhu. (11 feb. 2017) Short maturity Asian options for the CEV model. Preprint. Arxiv:1702.03382 v1 [q-fin.pr]. 